\title{
WIENER KLINISCHE WOCHENSCHRIFT
}

\author{
The Middle European Journal of Medicine
}

120. Jahrgang / Heft 13-14 2008

\section{Editorial}

Wien Klin Wochenschr (2008) 120/13-14: 387-389

DOI 10.1007/s00508-008-1024-3

(C) Springer-Verlag 2008

\section{Stress-Hyperglykämie - Einfluss auf Morbidität und Mortalität beim Akutpatienten}

\begin{abstract}
Die Stress-Hyperglykämie wurde bereits vor 130 Jahren von Claude Bernard in seinen Beobachtungen beim Blutungsschock erstmals ausführlich beschrieben [1]. Bis vor wenigen Jahren waren wir der Meinung, dass bei Intensivpatienten diese Stress-Hyperglykämie als adaptives Epiphänomen im Sinne einer physiologischen Gegenregulation zu akzeptieren ist und Blutzuckerwerte um $200 \mathrm{mg} / \mathrm{dl}$ sogar positive Effekte auf den Stoffwechsel des Intensivpatienten haben. $\mathrm{Zu}$ diesem gestörten Glukosestoffwechsel kommt es bei akuten Erkrankungen unabhängig von vorbestehenden Glukosestoffwechsel-Erkrankungen wie Diabetes mellitus. Dabei spielt die periphere Insulinresistenz, die durch eine Hyperinsulinämie, eine beeinträchtigte periphere Insulin-mediierte Glukoseaufnahme ins Muskelgewebe und eine gleichzeitig erhöhte hepatische Glukoneogenese charakterisiert ist, die zentrale Rolle [2].
\end{abstract}

Zahlreiche klinische Studien zeigen jedoch, dass diese Stress-Hyperglykämie nicht nur ein Epiphänomen darstellt, sondern negative Auswirkungen auf Morbidität und Mortalität aufweisen. Eine rezente Untersuchung bei 6187 Patienten auf einer Notfallaufnahme in Australien zeigte eindrucksvoll, dass der Blutzuckerwert bei der Aufnahme ein unabhängiger Prädiktor für die Mortalität darstellt [3]. Je höher der Blutzuckerspiegel war, umso höher war die Mortalität. Pro Anstieg des Blutzuckerspiegels um 1 mmol/1 stieg die Hasard-Ratio zu versterben signifikant um 1,04 an. Auffallend war aber vor allem, dass dieser Zusammenhang nur bei jenen Patienten bestand, die keinen vorbestehenden Diabetes mellitus hatten, nicht jedoch bei jenen Patienten mit bekanntem Diabetes mellitus.

Auch für spezielle Krankheitsbilder konnten ähnliche Zusammenhänge zwischen Blutzuckerspiegel und Morbidität beziehungsweise Mortalität gezeigt werden. Eine Metaanalyse ergab für den akuten Myokardinfarkt eine sehr enge Korrelation zwischen dem Ausmaß der StressHyperglykämie und der Entwicklung eines kardiogenen Schock, Herzversagens und erhöhter Spitalsmortalität [4]. Auch bei kardiochirurgischen Patienten ist eine perioperative Hyperglykämie mit einer erhöhten Mortalität assoziiert [5]. Ähnliche Ergebnisse zeigen sich auch bei Trauma-Patienten, bei denen die Hyperglykämie mit einer erhöhten Mortalität, verlängerter Liegedauer und erhöhter Infektionsrate assoziiert ist [6]. Auch bei Schlaganfallpatienten bewirkt eine Hyperglykämie ein höheres Risiko $\mathrm{zu}$ versterben und bei jenen Patienten, die überleben, ist die neurologische Rehabilitation umso schlechter, je ausgeprägter in der Akutphase die Hyperglykämie war [7].

In dieser Ausgabe der Wiener Klinischen Wochenschrift wird nun erstmals der Zusammenhang zwischen Vorhofflimmern bei akutem Myokardinfarkt und einer Stress-Hyperglykämie beschrieben [8]. Die Autoren führten eine retrospektive Analyse bei 543 Patienten mit akutem Myokardinfarkt durch und zeigten eindrucksvoll, dass eine Stress-Hyperglykämie mit einer erhöhten Prävalenz von Vorhofflimmern assoziiert ist. Jene Patientengruppe mit einer Stress-Hyperglykämie über $8 \mathrm{mmol} / \mathrm{l}$ und Vorhofflimmern hatten eine 14.5 fach höhere Spitalsmortalität als jene Patientengruppe ohne Stress-Hyperglykämie und Vorhofflimmern. Während eine Stress-Hyperglykämie in einer multivariaten Regressionsanalyse ein unabhängiger Prädiktor für eine erhöhte Mortalität war, stellte hingegen Vorhofflimmern keinen unabhängigen Risikofaktor dar.

Diese Ergebnisse werfen drei wichtige Fragen auf:

1. Warum ist beim akuten Myokardinfarkt das Auftreten einer nicht kardialen Nebenwirkung wie die Stress-Hyperglykämie im Gegensatz zu einer typischen kardialen Nebenwirkung wie das Vorhofflimmern ein unabhängiger Risikofaktor für eine erhöhte Mortalität?

2. Ist die Höhe der Stress-Hyperglykämie Folge oder Ursache für den Schweregrad des Myokardinfarktes und die erhöhte Mortalität?

3. Kann durch eine Senkung der Blutzuckerwerte im Rahmen einer Insulintherapie die Prognose des Myokardinfarktes verbessert werden?

Die Beantwortung der ersten Frage liegt möglicherweise in den negativen systemischen Effekten der Hyperglykämie auf unterschiedliche Zellsysteme. So konnten mehrere experimentelle und klinische Studien zeigen, dass eine Hyperglykämie negative Auswirkungen auf Endothelzellen, Neuronen, immunkompetente Zellen, Hepatozyten oder Alveolarzellen aufweist [9]. Wir wissen inzwischen, dass eine Hyperglykämie toxische Auswirkungen auf die Mitochondrien ausübt, den oxidativen Stress erhöht, die Monozyten- und Makrophagenfunktion nachhaltig negativ beeinflussen kann und durch Störung der Gerinnungskaskade zu einer Hyperkoagulopathie führen kann. 
Diese zahlreichen negativen systemischen Effekte erklären auch, warum eine Stress-Hyperglykämie so unterschiedliche Organsysteme wie Hirn, Niere, Herz oder Lunge nachhaltig negativ beeinflussen können. Es ist daher auch nachvollziehbar, dass im Rahmen eines akuten Myokardinfarktes das Auftreten einer Stress-Hyperglykämie einen wesentlich dramatischeren Effekt auf die Mortalität aufweist als das Auftreten von Vorhofflimmern. Die Ergebnisse der vorliegenden Studie in der Wiener Klinischen Wochenschrift stehen somit sehr gut im Einklang mit früheren Ergebnissen die eine enge Korrelation zwischen dem Ausmaß der Stress-Hyperglykämie und der Entwicklung eines kardiogenen Schocks und Herzversagens zeigten [4].

Mehrere Studien legen nahe, dass das Ausmaß einer Stress-Hyperglykämie vorwiegend eine unspezifische Folge eines Akutereignisses darstellt [3]. Auch Daten aus der eigenen Arbeitsgruppe zeigen bei Intensivpatienten, dass je höher der Schweregrad der Erkrankung gemessen am APACHE III Score ist, umso ausgeprägter ist die Beeinträchtigung der peripheren Insulinresistenz im Rahmen einer euglykämen hyperinsulinämen Clamp-Technik [10]. Trotz dieser Datenlage darf auch die Hypothese, dass die Hyperglykämie selbst für die erhöhte Mortalität prädisponierend ist, nicht außer Acht gelassen werden. Dies wir durch die Tatsache unterstützt, dass die Hyperglykämie per se eine Aktivierung zahlreicher Inflammationsparameter und Zytokine, eine Beeinträchtigung der Leukozytenfunktion und letztendlich zu einer erhöhten Infektionsrate führen kann [3]. Diese systemische inflammatorische Aktivierung führt zu einer Beeinträchtigung verschiedenster Organsysteme und könnte damit erklären, warum bei den unterschiedlichsten Akuterkrankungen die Stress-Hyperglykämie eine Verschlechterung der Morbidität und auch Mortalität bewirkt.

Die derzeit am meisten kontrovers diskutierte Frage ist, ob eine Therapie der Stress-Hyperglykämie durch Insulin diese negativen Auswirkungen kompensieren kann und damit eine Insulintherapie die Prognose bei akuten Erkrankungen verbessern kann. $\mathrm{Zu}$ diesem Thema wurde von Malmberg bereits vor mehr als 10 Jahren eine prospektive, randomisierte Studie bei 620 Patienten mit akutem Myokardinfarkt publiziert [11]. In jener Patientengruppe, die eine intensivierte Insulintherapie erhielt, kam es zu einer signifikanten Mortalitätsreduktion um $11 \%$. Malmberg berechnete aufgrund seiner Ergebnisse, dass lediglich 9 Patienten mit akutem Myokardinfarkt mit Insulin behandelt werden müssen um einen Todesfall durch die intensivierte Insulintherapie zu verhindern.

In den letzten Jahren wurden drei weitere große, prospektive, kontrollierte Studien publiziert [12-14]. Die ersten beiden Studien von Grete Van den Berghe (Leuven I und Leuven II Studie) zeigten eindrucksvoll, dass eine intensivierte Insulintherapie mit dem Ziel einer Normoglykämie (Blutzuckerzielbereich 110 bis $80 \mathrm{mg} / \mathrm{dl}$ ) zu einer Reduktion der Morbidität und zu einer Senkung der Mortalität führt [12, 13]. Vor allem jene Patienten die länger auf der Intensivstation aufgenommen waren (>3 Tage) profitierten am meisten von der strengen Normoglykämie unter der Intensivierten Insulintherapie, obwohl diese Patientengruppe die höchste Hypoglykämierate aufwies.
Auffallend in diesen Studien war auch die Tatsache, dass nicht nur die Mortalität gesenkt werden konnte sondern auch die Morbidität verschiedenster Organfunktionen deutlicher niedriger war als in der Kontrollgruppe. Es konnte sowohl das akute Nierenversagen, die Entwicklung einer Critical Illness Polyneuropathie, die Sepsisrate, eine Leberdysfunktion, als auch die Beatmungsdauer signifikant gesenkt werden [12].

Dass die klinische Umsetzung einer intensivierten Insulintherapie in der täglichen Praxis äußerst schwierig, ja sogar oft nicht ausreichend möglich ist und mit einer deutlich erhöhten Rate an Hypoglykämien einhergeht, wissen wir aus zahlreichen Studien [15]. In der kürzlich publizierten VISEP-Studie, an der insgesamt 18 Zentren in Deutschland teilnahmen, wurden insgesamt $488 \mathrm{~Pa}$ tienten (247 Patienten mit intensivierter Blutzuckereinstellung zwischen 80 und $110 \mathrm{mg} / \mathrm{dl}$ und 241 Patienten mit konservativer Blutzuckereinstellung zwischen 180$200 \mathrm{mg} / \mathrm{dl}$ ) mit schwerer Sepsis oder septischem Schock eingeschlossen [14]. Im Rahmen einer Sicherheitsinterimsanalyse wurde die Studie vorzeitig abgebrochen, da bei fehlenden Unterschieden in den primären Studienendpunkten wie Mortalität, bei jenen Patienten mit einem intensivierten Insulinschema deutlich häufiger schwere Hypoglykämien auftraten, vor allem aber die geplanten Blutzucker-Zielwerte sowohl in der intensivierten Insulintherapiegruppe als auch in der konservativen Blutzuckergruppe deutlich verfehlt wurden.

Trotz dieser Studienergebnisse gilt die Intensivierte Insulintherapie nach wie vor als Standardtherapie bei Intensivpatienten, wobei in den nächsten Jahren zu klären sein wird, welche Blutzuckerspiegel mit der intensivierten Insulintherapie tatsächlich angestrebt werden sollen und ob dies für alle Patienten einheitlich gilt oder zwischen verschiedenen Patientengruppen unterschiedliche Zielwerte sinnvoll sind.

Die Arbeit von Koracevic und Mitarbeitern in der vorliegenden Ausgabe der Wiener Klinischen Wochenschrift zeigt einmal mehr welche enorme Bedeutung die Stress-Hyperglykämie auf Morbidität und Mortalität beim Akutpatienten hat. Offen bleibt jedoch die Frage wie aggressiv erhöhte Blutzuckerwerte in der Akutphase gesenkt werden sollen und ob es Patientenkollektive gibt die mehr oder weniger von einer intensivierten Insulintherapie profitieren.

Christian Madl, Ulrike Holzinger, Reinhard Kitzberger, Joanna Warszawska

\section{Literatur}

1. Bernard C (1878) Leçons sur les phénomènes de la vie communs aux animaux et aux végétaux. J.B. Bailliere et Fils; Volume 1, pp 564

2. McCowen KC, Malhotra A, Bistrian BR (2001) Stressinduced hyperglycaemia. Crit Care Clin 17: 107-124

3. Cheung NW, Li S, Ma G, Crampton R (2008) The relationship between admission bloodglucose levels and hospital mortality. Diabetologia 51: 952-955

4. Capes SE, Hunt D, Malmberg K, et al (2000) Stresshyperglycemia and increased risk of death after myocardial infarction in patients with and without diabetes: a systematic overview. Lancet 355: 773-778 
5. Muhlstein JB, Anderson JL, Horne BD, et al (2003) Effect of fasting glucose levels on mortality rate in patients with and without diabetes mellitus and coronary artery disease undergoing percutaneous coronary intervention. Am J Heart 146: 351-358

6. Vanhorebeek OI, Languoche L, Van den Berghe G (2007) Tight blood glucose control with insulin in the ICU. Chest 132: $268-278$

7. Capes SE, Hunt D, Malmberg K, et al (2001) Stresshyperglycemia and prognosis of stroke in nondiabetic and diabetic patients: a systematic overview. Stroke 32: 24262432

8. Koracevic GP, Petrovic S, Damjanovic M, Stanojlovic T (2008) Association of stress hyperglycemia and atrial fibrillation in myocardial infarction. Wien Klin Wochenschr 120: 409-413

9. Van den Berghe G (2008) Insulin therapy in the intensive care unit should be targeted to maintain blood glucose between $4.4 \mathrm{mmol} / \mathrm{l}$ and $6.1 \mathrm{mmol} / \mathrm{l}$. Diabetologia 51: 911-915

10. Holzinger U, Kitzberger R, Fuhrmann V, Funk GC, Madl C, Ratheiser K (2007) Correlation of calculated indices of insulin resistance (QUICKI and HOMA) with the euglycaemic hyperinsulinaemic clamp technique for evaluating insulin resistance in critically ill patients. Eur J Anaesthesiol 24: 966-970

11. Malmberg K (1997) Prospective randomised study of intensive insulin treatment on long term survival after acute myocardial infarction in patients with diabetes mellitus. DIGAMI (Diabetes Mellitus, Insulin Glucose Infusion in Acute Myocardial Infarction) Study Group. BMJ 314: 1512-1515

12. Van den Berghe G, Wouters P, Weekers F, et al (2001) Intensive insulin therapy in critically ill patients. N Engl J Med 345: 1359-1367

13. Van den Berghe G, Wilmer A, Hermans G, et al (2006) Intensive insulin therapy in medical intensive care patients. N Engl J Med 354: 449-461

14. Brunkhorst FM, Engel C, Bloos F, et al (2008) Intensive insulin therapy and pentastarch resuscitation in severe sepsis. N Engl J Med 358: 125-1239

15. Nasraway SA (2007) Sitting on the horns of a dilemma: avoiding severe hypoglycemia while practicing tight glycemic control. Crit Care Med 35: 2435-2437

Korrespondenz: Ao. Univ. Prof. Dr. Christian Madl, Universitätsklinik für Innere Medizin III, Intensivstation 13H1, Medizinische Universität Wien, Währinger Gürtel 18-20, 1090 Wien, Österreich, E-mail: christian.madl@meduniwien.ac.at 